\title{
Multivariate Padé approximation for solving Fokker-Plank equations of fractional order
}

Veyis Turut

Department of Mathematics, Faculty of Arts and Sciences, Batman University, Batman, Turkey

Received: 14 May 2021, Accepted: 3 June 2021

Published online: 12 September 2021.

\begin{abstract}
In this paper, multivariate Padé approximation is applied to fractional power series solutions of Fokker-Plank equation with space- and time-fractional. As it is seen from comparisons, multivariate Padé approximation gives reliable solutions and numerical results.
\end{abstract}

Keywords: Multivariate Padé approximation, Fractional Fokker-Plank Equation, Variational Method.

\section{Introduction}

Fokker-Plank equation has been used in various areas such as, biophysics, neurosciences, population dynamic, polymer physics, nonlinear hydrodynamics, laser physics, pattern formation, surface, physics, psychology, marketing, engineering and plasma physics $[1,3]$. More details about about Fokker-Plank equation (FPE) can be found in $[4,6]$.

In recent times, unvariate and multivariate padé approximaton have been succesfully applied to various problems in physical and engineering sciences [7-13]. In this paper, multivariate Padé approximations were applied to the solutions ofFokker-Plank equation in the form [6]:

$$
\frac{\partial^{\alpha} u}{\partial t^{\alpha}}=\left[-\frac{\partial^{\beta}}{\partial x^{\beta}} A(x, t, u)+\frac{\partial^{2 \beta}}{\partial x^{2 \beta}} B(x, t, u)\right] u(x, t), t>0 \alpha>0 \quad \beta \leq 1
$$

where $\alpha$ and $\beta$ are parameters describing the order of the fractional time- and space-derivatives, respectively [6]. More details about equation (1) and basic definitions of properties of fractional calculus theory can be found in [6].

\section{Variational iteration method}

The basic concepts and principles of variational iteration method can be seen in [6, 14-18]. Odibat and Momani in [6] constructed the following iteration formula by using the basic concepts and principles of variational iteration method (VIM):

$$
u_{n+1}(x, t)=u_{n}(x, t)-\int_{0}^{t}\left(\frac{\partial^{\alpha}}{\partial \xi^{\alpha}} u_{n}(x, \xi)+\left[\frac{\partial^{\beta}}{\partial x^{\beta}} A\left(x, \xi, u_{n}\right)-\frac{\partial^{2 \beta}}{\partial x^{2 \beta}} B\left(x, \xi, u_{n}\right)\right] u_{n}(x, \xi)\right) d \xi
$$




\section{Multivariate Padé approximation}

Consider the bivariate function $f(x, y)$ with Taylor power series development

$$
f(x, y)=\sum_{i, j=0}^{\infty} c_{i j} x^{i} y^{j}
$$

around the origin [14]. The Padé approximation problem of order for $f(x, y)$ consists in finding polynomials

$$
\begin{aligned}
& p(x, y)=\sum_{k=0}^{m} A_{k}(x, y) \\
& q(x, y)=\sum_{k=0}^{n} B_{k}(x, y)
\end{aligned}
$$

such that in the power series $(f q-p)(x, y)$ the coefficients of $x^{i}$ and $y^{j}$ by solving the following equation system;

$$
\begin{aligned}
& \left\{\begin{array}{l}
C_{0}(x, y) B_{0}(x, y)=A_{0}(x, y) \\
C_{1}(x, y) B_{0}(x, y)+C_{0}(x, y) B_{1}(x, y)=A_{1}(x, y) \\
\vdots \\
C_{m}(x, y) B_{0}(x, y)+\cdots+C_{m-n}(x, y) B_{n}(x, y)=A_{m}(x, y)
\end{array}\right. \\
& \left\{\begin{array}{l}
C_{m+1}(x, y) B_{0}(x, y)+C_{m+1-n}(x, y) B_{n}(x, y)=0 \\
\vdots \\
C_{m+n}(x, y) B_{0}(x, y)+\cdots+C_{m}(x, y) B_{n}(x, y)=0
\end{array}\right.
\end{aligned}
$$

where $C_{k}=0$ if $k<0$. If the equations (6) and (7) are solved then the coefficients $A_{k}(k=0, \ldots, m)$ and $B_{k}(k=0, \ldots, n)$ are obtained. So polynomials (4) and (5) are found. Polynomials $p(x, y)$ and $q(x, y)$ are called Padé equations [19]. So the multivariate Padé approximant of order $(m, n)$ for $f(x, y)$ is defined as,

$$
r_{m, n}(x, y)=\frac{p(x, y)}{q(x, y)}
$$

\section{Applications and results}

In this section multivariate Padé series solutions of fractional Fokker-Plank equation with space- and time-fractional shall be illustrated by two examples. The full VIM solutions of examples can be seen in Odibat and Momani [6]

Example 1. Consider the linear space fractional FPE [6]

$$
\begin{gathered}
\frac{\partial u}{\partial t}=\left[-\frac{\partial^{\beta}}{\partial x^{\beta}} x+\frac{\partial^{2}}{\partial x^{2}} \cdot \frac{x^{2}}{2}\right] u(x, t), t>0 x>0, \\
u(x, 0)=x .
\end{gathered}
$$

where $0<\beta \leq 1$.

According to the iteration formulas (2) Odibat and Momani [6] obtained following solution by using variational iteration 
method,

$$
\begin{aligned}
u(x, t)= & +\left[\frac{3 x^{3-2 \beta}}{\Gamma(3-\beta) \Gamma(4-2 \beta)}-\frac{2 x^{2-\beta}}{\Gamma(3-\beta)}\right] t+\left[\frac{3 \Gamma(6-2 \beta) x^{5-4 \beta}}{2 \Gamma(4-2 \beta) \Gamma(6-4 \beta)}-\left(\frac{3 \Gamma(5-2 \beta)}{\Gamma(4-2 \beta)}+\frac{\Gamma(5-\beta)}{\Gamma(3-\beta)}\right) \frac{x^{4-3 \beta}}{\Gamma(5-3 \beta)}+\frac{2 \Gamma(4-\beta) x^{3-2 \beta}}{2 \Gamma(4-2 \beta) \Gamma(5-3 \beta)}\right] \frac{t^{2}}{2} \\
& +\left[\frac{3 \Gamma(6-2 \beta) \Gamma(8-4 \beta) x^{7-6 \beta}}{4 \Gamma(4-2 \beta) \Gamma(6-4 \beta) \Gamma(8-6 \beta)}-\left(\frac{3 \Gamma(6-2 \beta) \Gamma(7-4 \beta)}{2 \Gamma(4-2 \beta) \Gamma(6-4 \beta)}+\frac{3 \Gamma(5-2 \beta) \Gamma(7-3 \beta)}{2 \Gamma(4-2 \beta) \Gamma(5-3 \beta)}+\frac{3 \Gamma(5-\beta) \Gamma(7-3 \beta)}{2 \Gamma(3-\beta) \Gamma(5-3 \beta)}\right) \frac{x^{6-5 \beta}}{\Gamma(7-5 \beta)}\right. \\
& \left.+\left(\frac{\Gamma(4-\beta) \Gamma(6-2 \beta)}{\Gamma(3-\beta) \Gamma(4-2 \beta)}+\frac{3 \Gamma(5-2 \beta) \Gamma(6-3 \beta)}{\Gamma(4-2 \beta) \Gamma(5-3 \beta)}+\frac{\Gamma(5-\beta) \Gamma(6-3 \beta)}{\Gamma(3-\beta) \Gamma(6-4 \beta)}\right) \frac{x^{5-4 \beta}}{\Gamma(6-4 \beta)}-\frac{2 \Gamma(4-\beta) \Gamma(5-2 \beta) x^{4-3 \beta}}{\Gamma(3-\beta) \Gamma(4-2 \beta) \Gamma(5-3 \beta)}\right] \frac{t^{3}}{6}
\end{aligned}
$$

The exact solution of (9) is given as $u(x, t)=x e^{t}$ in [6]. VIM solution (11) for $\beta=1$ is obtained as

$$
u(x, t)=x+x t+0.500000000 x t^{2}+0.166666666 x t^{3}
$$

If the multivariate Padé approximation is applied to equation (12) for $m=2$ and $n=2$, according to the equation system (6) and (7) the following Padé equation is obtained;

$$
r_{2,2}(x)=\frac{1.00000000(2.00000000 t+6.000000026) x}{-4.000000024 t+1.000000012 t^{2}+6.000000026}
$$

VIM solution (11) for $\beta=0.25$ is obtained as

$$
\begin{aligned}
u(x, t)= & x+\left(0.9027033339 x^{2.5}-1.243503145 x^{1.75}\right) t+0.8274780560 x^{2.5} t^{2} \\
& -1.256022096 x^{3.25} t^{2}+0.4921875000 x^{4.00} t^{2}+0.2051598486 x^{5.5} t^{3} \\
& -0.7410817640 x^{4.75} t^{3}+0.7157464550 x^{4.00} t^{3}-0.3872420476 x^{3.25} t^{3} .
\end{aligned}
$$

So the multivariate Padé approximant of order $(13,2)$ for equation (14) is,

$$
r_{13,2}(x, t)=\frac{1.039330722\left(1+0.902703339 x^{3 / 2} t-1.2433503145 x^{3 / 4} t+0.8274780560 x^{3 / 2} t^{2}\right) x^{27 / 4} t^{2}}{-1.039330722 x^{23 / 4} t^{4}}
$$

VIM solution (11) for $\beta=0.75$ is obtained as

$$
\begin{aligned}
u(x, t)= & x+\left(2.256758335 x^{1.50}-1.765220243 x^{1.25}\right) t+1.692568752 x^{1.50} t^{2} \\
& -4.604847585 x^{1.75} t^{2}+3.281250002 x^{2.00} t^{2}+3.949327085 x^{2.50} t^{3} \\
& -7.567692255 x^{2.25} t^{3}+4.707004284 x^{2.00} t^{3}-1.165784199 x^{1.75} t^{3}
\end{aligned}
$$

So the multivariate Padé approximant of order $(5,2)$ for equation (16) is,

$$
\begin{aligned}
r_{5,2}(x, t)= & 1.000000000\left(3.327964170 x^{5 / 4} t^{2}-0.250005318 x t^{3}-2.197208468 x t^{2}\right. \\
& -0.502048564 x^{3 / 4} t^{3}+0.930134582 x^{3 / 4} t^{2}+2.949331452 x^{3 / 4} t+0.5456584678 \sqrt{x} t^{3} \\
& -0.221561445 \sqrt{x} t^{2}-0.973612653 \sqrt{x} t-0.9857951164 x^{1 / 4} t^{2}+0.5528406278 x^{1 / 4} t \\
& \left.+0.653444236 x^{1 / 4}+0.3833395592 t\right) x t /\left(( 1 + 2 . 2 5 6 7 5 8 3 3 5 \sqrt { x } t - 1 . 7 6 5 2 2 0 2 4 3 x ^ { 1 / 4 } t ) \left(0.5528406278 x^{1 / 4} t^{2}\right.\right. \\
& \left.\left.+0.367562383 x^{1 / 4} t^{3}+0.3833395592 t^{2}+1.333333333 \sqrt{x} t^{2}+0.653444236 x^{1 / 4} t\right)\right) .
\end{aligned}
$$


Example 2. Consider the linear space-and time-fractional FPE [6]

$$
\frac{\partial^{\alpha} u}{\partial t^{\alpha}}=\left[-\frac{\partial^{\beta}}{\partial x^{\beta}}\left(\frac{x}{6}\right)+\frac{\partial^{2}}{\partial x^{2}} \cdot\left(\frac{x^{2}}{12}\right)\right] u(x, t), \quad t>0 x>0
$$

where $\alpha>0, \beta \leq 1$, subject to initial condition

$$
u(x, 0)=x^{2}
$$

According to the iteration formulas (2) Odibat and Momani [6] obtained following solution by using variational iteration method,

$$
\begin{aligned}
u(x, t)= & x^{2}+2\left[\frac{2 x^{4-2 \beta}}{\Gamma(5-2 \beta) \Gamma(4-2 \beta)}-\frac{x^{3-\beta}}{\Gamma(4-\beta)}\right] t \\
& +\left[\frac{\Gamma(5-\beta) x^{4-2 \beta}}{6 \Gamma(4-\beta) \Gamma(5-2 \beta)}-\left(\frac{\Gamma(6-2 \beta)}{3 \Gamma(5-2 \beta)}+\frac{\Gamma(6-\beta)}{12 \Gamma(4-\beta)}\right) \frac{x^{5-3 \beta}}{\Gamma(6-3 \beta)}+\frac{\Gamma(7-2 \beta) x^{6-4 \beta}}{6 \Gamma(5-2 \beta) \Gamma(7-4 \beta)}\right] \frac{t^{2}}{2} \\
& -\left[\frac{2 x^{4-2 \beta}}{\Gamma(5-2 \beta) \Gamma(4-\beta)}-\frac{x^{3-\beta}}{\Gamma(4-\beta)}\right] \frac{t^{2-\alpha}}{\Gamma(3-\alpha)}
\end{aligned}
$$

The exact solution of (18) is given as $u(x, t)=x^{2} e^{t / 2}$ in [6]. VIM solution (20) for $\alpha=1, \beta=1$ is obtained as

$$
u(x, t)=x^{2}+0.50000000000 x^{2} t+0.1250000000 x^{2} t^{2}
$$

If the multivariate Padé approximation is applied to equation (21) form $=2$ and $n=2$, according to the equation system (6) and (7) the following Padé equation is obtained;

$$
r_{2,2}(x, t)=\frac{16.00000000 x^{2}}{2.000000000 t^{2}-8.000000000 t+16.00000000}
$$

VIM solution for the equation (20) (when $\alpha=0.25, \beta=0.25$ ) is;

$$
\begin{aligned}
u(x, t)= & x^{2}+2 .\left(0.171943492 x^{3.50}-0.2260914810 x^{2.75}\right) t \\
& +0.02686617064 x^{3.50} t^{2}-0.04237771068 x^{4.25} t^{2}+0.01718749999 x^{5.00} t^{2} \\
& -0.6217515726\left(0.1719434921 x^{3.5}-0.2260914810 x^{2.75}\right) t^{1.75}
\end{aligned}
$$

So the multivariate Padé approximant of order $(20,2)$ for equation (23) is,

$$
r_{20,2}(x, t)=\frac{-0.01142892204 x^{9} t^{7 / 2}\left(1+0.343886984 x^{3 / 2} t-0.1405727339 x^{3 / 4} t^{7 / 4}\right)}{-0.01142892204 x^{7} t^{7 / 2}}
$$

VIM solution for the equation (20) (when $\alpha=0.75, \beta=0.75$ ) is;

$$
\begin{aligned}
u(x, t)= & x^{2}+1.203604445 t x^{2.50}-0.7845423298 x^{2.25} t+0.08149405096 x^{2.50} t^{2}-02620070549 x^{2.75} t^{2} \\
& +0.2187500000 x^{3.00} t^{2}-0.5311567327 x^{2.50} t^{1.25}+0.3462225004 x^{2.25} t^{1.25}
\end{aligned}
$$


So the multivariate Padé approximant of order $(14,2)$ for equation (25) is,

$$
\begin{aligned}
r_{14,2}(x, t)= & .203604445 x^{2}\left(0.294205291 x^{1 / 4} t^{7 / 4}+0.2256773047 \sqrt{x} t^{7 / 4}+0.7845423292 x^{3 / 4} t^{3 / 2}\right. \\
& +0.3541067967 x^{3 / 4} t^{11 / 4}+1.231013334 x t^{5 / 2}+4.279482468 x^{5 / 4} t^{9 / 4}+7.438575074 x^{3 / 2} t^{2} \\
& +0.05093024585 \sqrt{x} t^{3}-0.6666710128 x^{1 / 4} t^{3 / 2}-4.184225756 x t^{9 / 4}-9 c ̧ 697333776 x^{5 / 4} t^{2} \\
& -1.203612291 x^{3 / 4} t^{5 / 2}-0.2308165049 \sqrt{x} t^{11 / 4}+1.203604445 \sqrt{x} t^{3 / 2}+0.6518273781 x^{1 / 4} \sqrt{t} \\
& +2.266006192 \sqrt{x} t^{1 / 4}+1.203604445 x t^{3 / 2}+1.477044875 x^{1 / 4} t^{1 / 4}+\sqrt{x} \sqrt{t}+0.9091309677 x^{9 / 4} t^{3 / 4} \\
& +0.2615158147 \sqrt{x} t^{5 / 2}+3.160493825 x t^{2}+3.555555551+2.727375125 x t^{5 / 4}+12.36049784 x t \\
& +0.4248789308 \sqrt{t}+5.134784062 \sqrt{x}-2.3176008455 \sqrt{x} t^{5 / 4}-8.056910904 x^{3 / 4} t \\
& \left.+0.3462225004 x^{3 / 4} t^{7 / 4}\right) /\left(\left(1.203604445+1.448663660 \sqrt{x} t-0.9442786354 x^{1 / 4} t\right.\right. \\
& \left.+0.4167149404 x^{1 / 4} t^{5 / 4}\right)\left(0.6518273781 x^{1 / 4} \sqrt{t}+2.266006192 \sqrt{x} t^{1 / 4}+0.4248789308 \sqrt{t}\right. \\
& \left.\left.+1.477044875 x^{1 / 4} t^{1 / 4}+5.134784062 \sqrt{x}+\sqrt{x} \sqrt{t}\right)\right)
\end{aligned}
$$

If the numerical results are compared, following tables are obtained (Table 1, Table 2);

\section{Conclusion}

As it is seen from the examples, it can be said that obtained numerical results by using multivariate padé approximation are as accurate as possible. It may be concluded that multivariate padé approximation is very powerful and efficient in finding numerical solutions for wide classes of space-time fractional partial differential equations.

Table 1: Numerical results and comparisons for the example 4.1.

\begin{tabular}{|l|l|l|l|l|l|l|l|l|}
\hline$x$ & $t$ & $\beta=0.25$ & $\beta=0.75$ & \multicolumn{3}{l|}{$\beta=1.0$} \\
\hline & & $u_{V I M}$ & $u_{M P A}$ & $u_{V I M}$ & $u_{M P A}$ & $u_{V I M}$ & $u_{M P A}$ & $u_{\text {Exact }}$ \\
\hline 0.01 & 0.01 & 0.00999615 & 0.00999615 & 0.00996680 & 0.00996680 & 0.01010050 & 0.01010050 & 0.01010050 \\
\hline 0.02 & 0.02 & 0.01997458 & 0.01997458 & 0.01986260 & 0.01986260 & 0.02040402 & 0.02040402 & 0.02040402 \\
\hline 0.03 & 0.03 & 0.02992365 & 0.02992366 & 0.02969220 & 0.02969220 & 0.03091363 & 0.03091363 & 0.03091363 \\
\hline 0.04 & 0.04 & 0.03983396 & 0.03983402 & 0.03946272 & 0.03946272 & 0.04163242 & 0.04163242 & 0.04163243 \\
\hline 0.05 & 0.05 & 0.04969749 & 0.04969767 & 0.04918157 & 0.04918157 & 0.05256354 & 0.05256355 & 0.05256355 \\
\hline 0.06 & 0.06 & 0.05950768 & 0.05950768 & 0.05885607 & 0.05885607 & 0.06371016 & 0.06371018 & 0.06371019 \\
\hline 0.07 & 0.07 & 0.06925691 & 0.06925796 & 0.06849338 & 0.06849338 & 0.07507550 & 0.07507554 & 0.07507557 \\
\hline 0.08 & 0.08 & 0.07894107 & 0.07894317 & 0.07810044 & 0.07810044 & 0.08666282 & 0.08666291 & 0.08666296 \\
\hline 0.09 & 0.09 & 0.08855476 & 0.08855864 & 0.08768402 & 0.08768402 & 0.09847543 & 0.09847559 & 0.09847568 \\
\hline 0.1 & 0.1 & 0.09809360 & 0.09810033 & 0.09725069 & 0.09725069 & 0.11051666 & 0.11051693 & 0.11051709 \\
\hline
\end{tabular}

\section{Competing interests}

The authors declare that they have no competing interests.

\section{Authors' contributions}

All authors have contributed to all parts of the article. All authors read and approved the final manuscript. 
Table 2: Numerical results and comparisons for the example 4.2.

\begin{tabular}{|l|l|l|l|l|l|l|l|l|}
\hline$x$ & $t$ & \multicolumn{2}{l|}{$\alpha=0.25, \beta=0.25$} & \multicolumn{2}{l|}{$\alpha=0.75, \beta=0.75$} & \multicolumn{2}{l|}{$\alpha=1.0, \beta=1.0$} \\
\hline & & $u_{V I M}$ & $u_{M P A}$ & $u_{V I M}$ & $u_{M P A}$ & $u_{V I M}$ & $u_{M P A}$ & $u_{\text {Exact }}$ \\
\hline 0.01 & 0.01 & 0.00009998 & 0.00009998 & 0.00009989 & 0.00009989 & 0.00010050 & 0.00010050 & 0.00010050 \\
\hline 0.02 & 0.02 & 0.00039981 & 0.00039981 & 0.00039916 & 0.00039917 & 0.00040402 & 0.00040401 & 0.00040402 \\
\hline 0.03 & 0.03 & 0.00089914 & 0.00089914 & 0.00089739 & 0.00089741 & 0.00091360 & 0.00091360 & 0.00091360 \\
\hline 0.04 & 0.04 & 0.00159751 & 0.00159751 & 0.00159434 & 0.00159441 & 0.00163232 & 0.00163231 & 0.00163232 \\
\hline 0.05 & 0.05 & 0.00249429 & 0.00249430 & 0.00248993 & 0.00249010 & 0.00256328 & 0.00256328 & 0.00256328 \\
\hline 0.06 & 0.06 & 0.00358876 & 0.00358880 & 0.00358423 & 0.00358461 & 0.00370962 & 0.00370961 & 0.00370963 \\
\hline 0.07 & 0.07 & 0.00488010 & 0.00488018 & 0.00487746 & 0.00487818 & 0.00507450 & 0.00507449 & 0.00507453 \\
\hline 0.08 & 0.08 & 0.00636736 & 0.00636752 & 0.00636994 & 0.00637120 & 0.00666112 & 0.00666111 & 0.00666118 \\
\hline 0.09 & 0.09 & 0.00804952 & 0.00804983 & 0.00806213 & 0.00806419 & 0.00847270 & 0.00847269 & 0.00847282 \\
\hline 0.1 & 0.1 & 0.00992548 & 0.00992601 & 0.00995456 & 0.00995778 & 0.01051250 & 0.01051248 & 0.01051271 \\
\hline
\end{tabular}

\section{References}

[1] Frank T D, 2004, Physica A, 331: 391.

[2] Acebron J A, 2005, et al., Rev. Mod. Phys., 77: 137.

[3] Frank T D, 2005, Nonlinear Fokker-Planck Equations: Fundamentals and Applications, Springer, Berlin.

[4] Risken H, 1988, The Fokker-Planck Equation, Springer, Berlin,

[5] Liu F, Anh V, Turner I, 2004, J. Comp. Appl. Math., 166 (1): 209.

[6] Odibat Z, Momani S, 2007, Numerical solution of Fokker-Plank Equation with space- and time-fractional derivatives, Physics Letters A, 369: 349-358.

[7] Celik E, Karaduman E, Bayram M, 2003, Numerical Solutions of Chemical Differential- Algebraic Equations, Applied Mathematics and Computation,139(2-3): 259-264.

[8] Celik E, Bayram M, 2004, Numerical solution of differential-algebraic equation systems and applications, Applied Mathematics and Computation, 154(2): 405-413.

[9] Turut V, Guzel N, 2012, Comparing Numerical Methods for Solving Time-Fractional Reaction- Diffusion Equations, ISRN Mathemtical Analysis, Doi:10.5402/2012/737206.

[10] Turut V, Guzel N, 2013, Multivariate padÃ@ $\odot$ approximation for solving partial differential equations of fractional order”,Abstract and Applied Analysis, Doi:10.1155/2013/746401.

[11] Turut V, Celik E, Yigider M, 2011, Multivariate pade approximation for solving partial differential equations (PDE), International Journal For Numerical Methods In Fluids, 66 (9):1159-1173.

[12] Turut V, 2012, Application of Multivariate padÃ@ approximation for partial differential equations, Batman University Journal of Life Sciences, 2 (1): 17-28.

[13] Turut V, 2013, Numerical approximations for solving partial differential equations with variable coefficients, Applied and Computational Mathematics, 2 (1): 19-23.

[14] He J H, 1998, Approximate analytical solution for seepage flow with fractional derivatives in porous media, Comput. Methods Appl. Mech. Eng., 167: 57-68.

[15] He J H, 1999, Variational iteration method-a kind of non-linear analytical technique: some examples, Int. J. Non-Linear. Mech., 34: 699-708.

[16] He J H, 2007, Variational iteration method-some recent results and new interpretations, J. Comput. Appl. Math., 207: 3-17.

[17] He J H, Wu X H, 2007, Variational iteration method: new development and applications, Comput. Math. Appl., 54: 881-894.

[18] He J H, Wu G-C, Austin F, 2010, The variational iteration method which should be followed, Nonlinear Sci. Lett. A, 1: 1-30.

[19] Cuyt A, Wuytack L, 1987, Nonlinear Methods in Numerical Analysis, Elsevier Science Publishers B.V., Amsterdam. 